\title{
La francophonie en Turquie de l'Empire à nos jours
}

\section{Ekrem Aksoy}

\section{(2) OpenEdition \\ Journals}

Édition électronique

URL : https://journals.openedition.org/dhfles/138

DOI : $10.4000 /$ dhfles. 138

ISSN : 2221-4038

\section{Éditeur}

Société Internationale pour l'Histoire du Français Langue Étrangère ou Seconde

Édition imprimée

Date de publication : 1 janvier 2007

Pagination : 57-66

ISSN : 0992-7654

Référence électronique

Ekrem Aksoy, "La francophonie en Turquie de l'Empire à nos jours », Documents pour l'histoire du français langue étrangère ou seconde [En ligne], 38/39 | 2007, mis en ligne le 18 décembre 2010, consulté le 27 mai 2021. URL : http://journals.openedition.org/dhfles/138 ; DOI : https://doi.org/ 10.4000/dhfles. 138

Ce document a été généré automatiquement le 27 mai 2021. 


\title{
La francophonie en Turquie de l'Empire à nos jours
}

\author{
Ekrem Aksoy
}

1 La diffusion du français hors de France et l'histoire de la francophonie ont attiré et attirent l'attention de plusieurs auteurs français et turcs. Selon des auteurs français, parmi les raisons qui ont déterminé l'hégémonie de la culture française dans le monde, se trouvent en tête la situation géographique et l'importance démographique de la France, les qualités de la langue française telle la clarté, la supériorité de l'art et de la littérature français. Les auteurs turcs y ajoutent l'amitié traditionnelle turco-française et les efforts culturels des Français au Proche-Orient. Chacune de ces causes doit avoir joué un rôle plus ou moins grand, mais elles sont insuffisantes pour expliquer l'expansion de la francophonie dans le monde et plus particulièrement en Turquie. Car la plupart de ces auteurs minimisent la prépondérance politique et militaire et la puissance économique de la France au XVII ${ }^{\mathrm{e}}$ siècle, puisqu'il

n'existe aucun synchronisme, aucune connexion nécessaire entre l'hégémonie politique et la culturelle [...]. L'expansion maximale de la langue ne correspond pas à l'époque des victoires du Grand Roi, mais à la décadence française sur le continent [...]. C'est sous le règne de Louis XV, si désastreux au point de vue militaire et colonial que la langue française a poussé le plus loin ses conquêtes ${ }^{1}$.

Louis Réau, à qui nous devons un des meilleurs ouvrages dans ce domaine, a bien raison dans ces constatations. Mais il ne faut pas oublier que, selon N. Danilevski, c'est au mois de juin que les jours sont les plus longs, mais c'est en juillet et en août qu'il fait le plus chaud et que les promesses du printemps se réalisent en été ou en automne ${ }^{2}$. Cela signifie que la France de Louis XV a récolté ce que la France de Richelieu, de Mazarin et Louis XIV avait semé. C'est pourquoi il sera question dans cette communication en premier lieu des relations politiques, économiques, religieuses et culturelles entre la France et la Turquie et, en second lieu, des fruits que ces relations ont portés le moment venu.

3 Les Turcs ont embrassé au cours de leur histoire d'abord la civilisation extrême orientale et ensuite, après avoir adopté l'Islam, la civilisation orientale. Quant aux Turcs ottomans, dans leur grande marche vers l'ouest, ils ont rencontré d'abord les 
Arméniens et les Grecs, ensuite d'autres peuples balkaniques; ils sont entrés en relation avec les pays méditerranéens et européens et ils ont fini par décider de s'intégrer à la civilisation occidentale qu'ils ont connue par l'intermédiaire de la France ou par la fenêtre française comme on ledit en Turquie. D'abord leur culture a attiré l'attention en Europe: en sont pour preuves les Écoles des jeunes de langues, les tragédies du XVII siècle dont l'action se passe dans l'Empire ottoman, les turqueries au XVIII siècle et Charles Verney (né en 1842 à Paris), poète français qui a appris le turc tout seul sans avoir mis le pied aux pays des Turcs, et qui a composé un divan en turc ${ }^{3}$.

Par la suite, c'est la France qui a exercé son influence culturelle en Turquie dans la période du déclin et du démembrement de l'Empire ottoman, et cela jusqu'à la Deuxième Guerre mondiale, de sorte que le français est devenu la langue seconde en Turquie alors que l'Europe n'était plus française comme au XVIII ${ }^{e}$ siècle. Nos défaites nous ont valu, écrit Jean Bourguignon dans sa préface à la Fièvre d'Âme d'Abdullah Cevdet publiée en 1901,

comme un déchaînement de haines et d'injures: on a pu voir un historien, Mommsen, comparer notre littérature aux «eaux bourbeuses de la Seine », et un critique, Matthew Arnold, affirmer gravement qu'en 1870 ce qui avait succombé avec la France, c'était « la déesse Lubricité "4.

Cela signifie qu'au XVIII ${ }^{\mathrm{e}}$ siècle, lorsque l'Europe était française, l'Empire ottoman ne l'était pas du tout et que Osman n'a pas plaidé en français les droits de son sultan en 1774 pendant les négociations de paix avec la Russie, comme le dit Pierre-Henri Simon ${ }^{5}$. En effet, le texte du traité de Küçük Kaynarca (Kutchuk-Kaïnardji) est en turc, en russe et en italien, mais non en français; les Russes l'ont traduit en français plus tard, et il se trouve que des historiens ont utilisé cette traduction.

\section{Montée de la prépondérance du français}

6 Ici se posent deux questions qui se complètent : premièrement, pourquoi, en Turquie, la culture française plutôt qu'anglaise ou allemande et pourquoi cet écart temporel entre la francisation des autres pays de l'Europe et celle de la Turquie ? À ce sujet, nous avons formulé l'hypothèse de l'action convergente de deux facteurs :

7 1. Malgré ses efforts déployés surtout au XVII ${ }^{\mathrm{e}}$ siècle, la France n'avait pas pu établir son hégémonie en Europe; elle avait dû se contenter de sa place de puissance après l'Angleterre et même après l'Autriche. En plus, l'Angleterre, première puissance, avait stoppé l'expansion de la France dans le Nouveau Monde. C'est la raison pour laquelle cette dernière a dû continuer son commerce par les routes traditionnelles qui passaient par des pays sous domination ottomane. D'autre part, la France, puissance de second ordre, et l'Empire ottoman en déclin devaient s'allier puisqu'ils avaient comme ennemi commun la maison d'Autriche. En effet, sauf pendant la campagne d'Égypte de Napoléon et sauf le cas d'une coalition européenne ou chrétienne, les deux pays ont souvent pris place dans le même camp jusqu'à l'entrée de l'Allemagne dans la scène politique internationale.

2. Les musulmans ottomans n'apprenaient pas du tout les langues européennes, langues des infidèles selon eux, surtout au moment de leur apogée et dans leur période de stagnation. Ils se servaient, dans leurs relations avec l'Europe, de traducteurs et d'interprètes appartenant à des communautés non musulmanes, plus particulièrement des Grecs phanariotes jusqu'aux années 1820-1830, puis des Arméniens. Par ailleurs, ils 
n'avaient pas du tout besoin d'apprendre ces langues, car les Occidentaux qui faisaient le commerce dans les échelles du Levant, avaient fondé des Écoles de jeunes de langues destinées à former des interprètes pour les besoins de la diplomatie et du commerce (Venise en 1551, France en 1669), et dans lesquelles on apprenait le latin, l'arabe, le persan, l'arménien et le turc, les langues véhiculaires, à côté de l'italien, du bassin méditerranéen jusqu'au XIXe siècle. Avec le commencement des défaites militaires, ils ont pensé à prendre les méthodes et techniques occidentales pour arrêter le recul de leurs armées devant celles de leurs ennemis; ce qui confirme l'hypothèse de la diffusion quant aux changements dans les cultures, selon laquelle l'homme, par essence imitateur, conservateur et enclin à la paresse, emprunte à autrui ce dont il a besoin au lieu de le créer lui-même.

9 Tout cela a donné lieu dans l'Empire ottoman à la prépondérance de la France, prépondérance politique à partir $\mathrm{du} \mathrm{XVI}^{\mathrm{e}}$ siècle et économique après les traités d'Utrecht (1713) et de Rastadt (1714) qui ont mis fin à la guerre de succession d'Espagne et par lesquels la France faisait d'importantes concessions maritimes aux Anglais. Dans ces conditions, les Ottomans, pour remédier à leur recul, ne pouvaient demander que l'aide de la France qui avait, à son tour, besoin des Turcs, et avec laquelle ils avaient le plus de relations politiques, économiques, religieuses et culturelles, facteurs qui favorisent l'expansion d'une culture à l'étranger.

10 Les conditions historiques ont donc obligé la France et la Turquie à devenir des alliées politiques et des partenaires économiques naturels. En effet, la France détenait les trois cinquièmes du commerce total de l'Empire ottoman avec l'Europe, contre un cinquième pour les Anglais, dans les quelques décennies qui ont précédé la Révolution française ${ }^{6}$. Les relations culturelles et religieuses ont aussi contribué au rapprochement des deux pays. Car le Sultan avait des sujets catholiques romains, arméniens ou autres qui seront par la suite les protégés de la France. Et, bien que leur objectif initial ne soit pas d'enseigner le français aux Ottomans musulmans, on voit dès le $\mathrm{XVI}^{\mathrm{e}}$ siècle dans l'Empire la création d'écoles françaises aux portes desquelles les musulmans finiront par frapper. Parmi ces écoles, celle des enfants de langues ou jeunes de langues, destinée à former des drogmans pour les besoins de la diplomatie et du commerce dans les échelles du Levant et qui se trouve à l'origine de l'INALCO actuel, a une histoire très significative parce que

sa fondation avait dans le principe une autre destination. Une dame avait légué dix mille livres applicables à l'entretien de dix jeunes Arméniens, qui, après avoir reçu à Paris leur éducation chez les Jésuites, devaient repasser en Turquie pour y faire la mission ou y seconder les Missionnaires ${ }^{7}$.

11 Les relations politiques, économiques, religieuses et culturelles entre la France et la Turquie porteront leurs fruits vers la fin du XVIII ${ }^{e}$ siècle : les signes en sont la demande d'experts français pour les réformes de l'armée et la création d'écoles sur le modèle européen auxquelles la France apportera sa contribution. Dans ces écoles, des enseignants français donnaient des cours en français, que les interprètes traduisaient en langue turque. Le collège des enfants de langues de Saint Georges fondé en 1745 à Istanbul par les capucins français sur la demande du roi des Deux-Siciles et qui enseignait le turc, a cessé de fonctionner probablement en 1783 et l'École des jeunes de langue française perdra de son importance à la même époque, alors qu'en 1793, à l'École du Génie militaire le français devient une matière obligatoire. On peut appeler cette longue période qui va jusqu'aux années 1820 , la période de l'introduction de la culture française en Turquie. 


\section{L'expansion du français} Bureau de traduction par la Sublime Porte pour former des fonctionnaires musulmans connaissant le français et qui devaient prochainement remplacer les interprètes grecs phanariotes au service de l'État, parce que l'insurrection grecque avait entraîné la suspicion des Ottomans à l'égard de cette communauté pendant un certain temps.

13 Turquie. Cette école a conduit, d'une part, les éléments turcs à prendre conscience de leur propre identité, parce que le cours d'histoire commençait avec ce fameux nos ancêtres les Gaulois et que les élèves se sont bientôt rendu compte que cela n'était pas du tout vrai à Istanbul ; et de l'autre, elle a contribué à la diffusion de la culture française en Turquie.

14 Le français devient, dans cette période, un moyen de communication tant avec l'étranger qu'à l'intérieur du pays. Car les divers groupes ethno-religieux qui constituaient l'Empire n'avaient pas de langue commune. Ce problème s'est posé par exemple au Parlement ottoman inauguré le 19 mars 1877. Selon la Constitution, la langue officielle était le turc, mais cette Assemblée réunissait environ sept cents députés de onze confessions différentes venus de toutes les régions. On y utilisait seize langues, sans parler des grandes différences entre le turc parlé et l'osmanli écrit et entre les dialectes. Cette situation linguistique de l'Empire est l'une des causes de l'emploi du français dans certaines institutions de première importance. En effet, la langue de travail du ministère des Affaires étrangères était le français de 1854 jusqu'en 1910. Qui plus est, des diplomates ottomans et des employés de ce ministère parlaient français entre eux, car des sujets qui ne savaient pas le turc pouvaient devenir fonctionnaires d'État conformément au principe de l'égalité imposé à la Sublime Porte par l'Europe. La Banque ottomane chargée de tenir lieu de banque centrale, la Régie des tabacs, les Chemins de fer et les Dettes publiques, chacun un véritable État dans l'État, et le Conseil international de la santé, utilisaient, comme le ministère des Affaires étrangères, le français qui était désormais langue d'enseignement, des sciences et de la presse. En effet, M. Sinan Kuneralp, ancien diplomate, éminent chercheur et propriétaire des Éditions ISIS, nous a rapporté, lors d'un colloque à l'université Hacettepe, qu'il avait répertorié jusque-là plus de 3500 livres en français, grands ou petits, publiés en Turquie de 1839 à 1922. Quant à Gérard Groc et Izzet Çağlar, ils nous informent que sont parus plus de 600 périodiques entièrement ou partiellement en français, dont la majorité écrasante entre 1868 et la fin de la Deuxième Guerre mondiale ${ }^{8}$. Et nombreux sont les Turcs qui ont composé des poèmes, des romans, des pièces de théâtre ... directement en français.

La dernière étape de cette évolution aurait sans aucun doute été l'adoption du français à qui on assignait le rôle de fusionner les diverses communautés ethniques et religieuses, comme langue officielle de la nation ottomane, qu'une partie de l'élite du pays projetait de créer pour arrêter le recul et la dislocation. C'est de cette élite qui constituerait le noyau de la nation ottomane que le français deviendrait la langue commune. En d'autres termes, la pax ottomana que l'Empire s'était donné la mission de faire régner sur les terres qu'il avait conquises, allait être remplacée par la pax francofona sous la pression des puissances occidentales. En effet, dans son observation 
sur l'article du mémoire adressé le 22 février 1867 par la France à la Sublime Porte concernant la création d'une université, le baron Prokesch, diplomate autrichien et internonce à Istanbul, annonce la fondation, entre autres écoles, du lycée de Galatasaray qui a réalisé la mixité ethno-confessionnelle et qui a ainsi permis à des sujets non musulmans d'accéder à de hautes fonctions de l'État. La fondation d'une université, écrit-il,

où les musulmans et les chrétiens (leur éducation préparatoire supposée) seraient admis, pourrait devenir, sans doute, un des puissants moyens de fusion. La difficulté sera de trouver des professeurs capables pour occuper les chaires, peutêtre les voudrait-on tirer de dehors; la diversité des idiomes se présentera d'abord comme un obstacle et frayera le chemin à l'introduction de la langue française comme langue générale de la civilisation et du progrès ${ }^{9}$.

\section{Le déclin}

Les hommes politiques et les intellectuels turcs se sont rendu compte que ces efforts pour la création d'une nation ottomane étaient voués à l'éche ${ }^{10}$. Car le nationalisme à l'européenne avait déjà gagné les peuples non musulmans de l'Empire. En effet la Première Guerre mondiale a mis fin à l'Empire ottoman, et la proclamation de la république à cette évolution du français qui a perdu en 1929 le monopole qu'il exerçait dans l'enseignement supérieur turc, sauf dans la Marine et l'École de Guerre. Depuis, le français est en recul rapide et continuel en Turquie, surtout depuis la Deuxième Guerre mondiale et il occupe aujourd'hui la troisième place après l'anglais et l'allemand et même la quatrième ou la cinquième après l'italien et l'espagnol. Mais son déclin rapide en Turquie ne peut pas être attribué à la seule montée de l'anglais qui a mis fin en 1815 à son hégémonie en Europe et qui a pris place à son côté en 1918 dans la diplomatie. Cela provient sûrement du fait que la France et la Turquie, qui s'étaient rapprochées à partir du XVI siècle, se sont très souvent trouvées dans des camps opposés, surtout à partir du dernier quart du XIX siècle, au moment même où la culture française avait atteint son apogée dans l'Empire ottoman. En effet, pendant la Première Guerre mondiale, la Turquie a dû combattre contre la France aux Dardanelles, en Cilicie et dans le sud-est anatolien. Ankara et Paris se sont affrontées à cause du sandjak d'Alexandrette à la veille de la Deuxième Guerre mondiale. Et de nos jours, bon nombre de problèmes politiques éloignent les deux pays l'un de l'autre. La nation arménienne, nation fidèle de l'Empire, qui avait contribué au développement de la francophonie en Turquie jusqu'à la fin du XIX siècle, est devenue un des grands obstacles politiques. La loi arménienne de 2001 et la proposition de loi visant à punirla négation du génocide adoptée en 2006 par l'Assemblée française ont fait perdre à la France non seulement les marchés mais aussi l'estime des Turcs, et elles ont tendu encore une fois les relations politiques, économiques et militaires sur lesquelles reposent les relations culturelles.

Les questions chypriote, kurde et même l'Union européenne à laquelle la Turquie a la vocation de s'intégrer alors que la France veut lui barrer fermement la porte, mettent en danger la francophonie qui faisait pourtant quelques efforts nouveaux mais insuffisants en Turquie pendant les cessez-le-feu à la suite des différends qui ne vont pas, heureusement, jusqu'à l'hostilité. En est un des exemples cette université Galatasaray, une des meilleures universités turques, qui continue la mission du lycée qui porte le même nom et qui nous a accueillis dans ses salons. 
Pour conclure, il faut ajouter que si les discordes entre Paris et Ankara continuent, la francophonie ne sera qu'un petit détail dans l'histoire de la culture turque à l'avenir, malgré l'existence d'une université, des deux départements francophones des universités Marmara et d'Antalya, des départements de français d'une dizaine d'universités et des lycées français dont l'enseignement est en français et de quelques lycées dits anatoliens où on apprend le français comme première langue étrangère, et malgré les efforts déployés par des hommes d'affaires turcs qui sont en relation avec des pays francophones, par des professeurs et des diplomates français et turcs qui font leur possible pour le maintien et pour la promotion de la francophonie en Turquie.

\section{NOTES}

1. Louis Réau [1938] et (1971), L'Europe française au siècle des Lumières, Paris, Albin Michel, p. 266.

2. Voir P. A. Sorokin, Bir Bunalım Çağında Toplum Felsefeleri (Social Philosophiesin Age of Crisis)(1972), traduction en turc par Mete Tuncay, Ankara, Bilgi Yayınevi, p. 68.

3. Voir Ibnül Emin Mahmut Kemâl Inal (1988), Son Asır Türk şairleri, IV. Cilt, Istanbul, Dergâh Yayınları, troisième édition, pp. 1983-1986.

4. Jean Bourguignon, «Présentation biographique», dans Abdullah Cevdet (Djevdet) (1901), Fièvre d'Âme, Vienne, Chez M. Frick, Libraire de la Cour Impériale et Royale, pp. XIII-XIV.

5. Voir Pierre-Henri Simon (1966), Diagnostic des lettres françaises contemporaines, Bruxelles, La Renaissance du Livre, p. 18.

6. Ali Ihsan Bağış (1985), «III George Döneminde Ingiltere'nin Osmanlı Imparatorluğundaki Ekonomi Siyaseti, 1760-1815 », dans Türk-Ingiliz Ilişkileri, 1583-1984, Ankara, Başbakanlık BasınYayın ve Enformasyon Müdürlüğü, Kurtuluş Ofset Basımevi, p. 44.

7. Henri Cordier (1911), Un Interprète du général Brune et la fin de l'École des jeunes de langues, Paris,

C. Klincksieck, p. 5.

8. Voir Gérard Groc et Izzet Çağlar (1985), La Presse française de Turquie de 1795 à nos jours : histoire et catalogue, Istanbul, Éditions ISIS.

9. Dans le Baron I. De Testa (1892), Recueil des traités de la Porte ottomane avec les puissances étrangères, Tome VII, Paris, Ernest Leroux éditeur, p. 423.

10. Voir Prof. Dr. Ercüment Kuran (1994), «Bir Türk Islahatçısı Olarak Küçük Sait Paşa (1840-1914)», dans Türkiye'nin Batıllış̧ması ve Millî Meseleler, Ankara, Türkiye Diyanet Vakfı Yayınları/135, 171. s.

\section{RÉSUMÉS}

Les Ottomans n'apprenaient pas du tout, lorsqu'ils étaient puissants, les langues européennes, langues des « infidèles » selon eux, et se servaient d'interprètes et de traducteurs appartenant à 
des communautés non musulmanes, plus particulièrement des Grecs phanariotes, dans leurs relations avec l'Europe. Le déclin commencé, la défaite et le recul de leurs armées devant les européennes les ont obligés à reconnaître les développements réalisés en Europe et, sous la pression des conditions historiques, la France et la Turquie ottomane ont dû se rapprocher l'une de l'autre. Les relations économiques, politiques, culturelles et religieuses au XVIII ${ }^{\mathrm{e}}$ siècle entre les deux pays ne porteront leurs fruits qu'au XIX ${ }^{\mathrm{e}}$ siècle, et on pensera à faire du français la langue de la «nation ottomane » qu'on a projeté de créer. Le français devient ainsi, à la fin du $\mathrm{XIX}^{\mathrm{e}}$ et au début du XX $\mathrm{XX}^{\mathrm{e}}$ siècle, langue d'élite en Turquie alors qu'il était en recul en Europe. Mais le français perdra le monopole d'être la seule langue enseignée dans l'enseignement supérieur en 1929, et sa suprématie au profit de l'anglais dans les années qui ont suivi la Deuxième Guerre mondiale.

The Ottomans, when strong, did not learn the European languages at all, language of the "infidels" and used interpreters and translators from the non-Muslim community, particularly from the Phanariote Greeks in their relations with Europe. When the decline started, the defeat and fall of their armies against the Europeans obliged them to recognize the developments realized in Europe. Under the pressure of the historical conditions, France and the Ottoman Turkey became closer. These relations bring fruit only in the $19^{\text {th }}$ century and French is thought to become the language of the "Ottoman nation". Consequently, at the end of the $19^{\text {th }}$ and the beginning of the $20^{\text {th }}$ century, French became the language of the elite in Turkey. At this time, it was in the zenith in Turkey whereas in Europe, it was in descent. French will lose the monopole to be the sole language trained in the higher education in 1929 and the supremacy to the profit of English in the years that followed the World War II.

\section{INDEX}

Keywords : Ottoman Turkey, history, European languages, decline, elite

Mots-clés : Turquie ottomane, histoire, langues européennes, déclin, élite

\section{AUTEUR}

\section{EKREM AKSOY}

Université Hacettepe, Turquie 